\title{
QUALIDADE: UMA REVISÃO DA LITERATURA NO ÂMBITO DE ENCONTROS DE MARKETING
}

CARPEJANI, Eduardo; AGUIAR, Wellington; ROSSI, Guilherme; TERRA, Natália; TROCCOLI, Irene Raguenet. Universidade Estácio de Sá, Mestrado em Administração e Desenvolvimento Empresarial. Av. Presidente Vargas, 642, 22. ${ }^{\circ}$ andar - Centro. CEP: 20071-001 - Rio de Janeiro - RJ. Tel: (21) 2206-9741/9743. E-mail: ppgadm.edu@estacio.br.

\section{Resumo}

O construto qualidade tem sido amplamente pesquisado na literatura acadêmica de Marketing voltada para a busca pelo entendimento dos fatores causadores do hiato entre o que espera o consumidor e o que é de fato percebido. O presente artigo traz levantamento bibliográfico relativo aos 20 artigos apresentados nos quatro Encontros de Marketing (EMAs) promovidos pela Associação Nacional dos Cursos de Pós-Graduação em Administração (Anpad), que continham a palavra qualidade no título. Verificou-se que os construtos subsidiariamente mais utilizados para suportar os estudos foram satisfação e lealdade/confiança/comprometimento e que, dentre os trabalhos analisados, raramente houve similaridade nos referenciais. Como sugestão para futuros estudos, propõe-se pesquisa semelhante no âmbito dos encontros da Anpad.

Palavras-chave: Revisão da literatura; Qualidade; Percepções; Comportamento do consumidor.

\begin{abstract}
The quality construct has been widely researched in the marketing literature focused on understanding the gap between what consumers expect and what is actually perceived. This paper presents a literature review of the 20 articles presented at the four Encontros de Marketing (EMAs) sponsored by Associação Nacional dos Cursos de Pós-Graduação em Administração (ANPAD), which mentioned the word quality in their titles. The alternative constructs most widely used to support the studies were satisfaction and loyalty / trust / commitment; and the studies that were analyzed seldom contained similar references. As a suggestion for future studies, a similar research in the context of the Encontros da ANPAD is proposed.
\end{abstract}

KeYwORDs: Literature review; Quality; Perceptions; Consumer behavior. 


\section{INTRODUÇÃo}

Os estudos acadêmicos de Marketing que versam sobre a percepção de qualidade por parte do consumidor, em sua ampla maioria, enfocamo mercado de serviços. De fato, a quantidade de monografias, de dissertações, de teses e de artigos científicos produzidos sobre a percepção de qualidade no consumo de serviços é infinitamente maior do que aquela que se observa para o caso de produtos físicos. Pode-se supor que tal se deva a dois fatores. $\mathrm{O}$ primeiro seria a grande contribuição que autores como Zeithaml, Bitner e Gremler (2011), Bitner (1992) e Lovelock e Wirtz (2006) garantiram à disciplina Marketing de Serviços, ao se lançar em pesquisas que deram origem a livros didáticos e a artigos acadêmicos de elevada qualidade. E estes, sem dúvida, contribuíram para despertar o interesse dos estudantes e pesquisadores de Administração de Empresas no estudo desse tema - principalmente aqueles estudantes e pesquisadores versados na abordagem quantitativa, a partir do esforço bem-sucedido de Zeithaml, Parasuraman e Berry $(1988,1990)$ na formatação de instrumento de medição da qualidade percebida em serviços, o Servqual.

O segundo fator que explicaria esse interesse tão disseminado seria a própria intangibilidade dos serviços. Enquanto uma das características que os distinguem dos produtos físicos, ela implica uma, por assim dizer, volatilidade que garante um certo charme desafiador à busca deste conhecimento - afinal, é possível se conhecer realmente a qualidade de algo tão abstrato quanto um serviço? - que parece retroalimentar as pesquisas que buscam esta resposta.

Uma vez que não limitado à área de serviços, este artigo busca expandir a baixa oferta de trabalhos de Marketing com foco em qualidade não restrita ao segmento terciário da Economia. Com ele, pretendese prover, àqueles que se interessem pelo tema percepção de qualidade de uma forma geral, uma amostra de encadeamentos lógicos tratados na área de Administração de Empresas a respeito deste assunto. Isso se fez por meio do mapeamento dos autores - e de suas concepções teóricas e práticas - citados nos referenciais teóricos dos artigos apresentados nos quatro Encontros de Marketing (EMAs) promovidos pela Associação Nacional dos Cursos de Pós-Graduação em Administração (Anpad) até o ano de 2010, que tenham trazido a palavra qualidade em seus títulos.

Além desta introdução, o presente artigo traz a metodologia do estudo, os resultados do levantamento bibliográfico, as conclusões e, finalmente, sugestões para futuros estudos.

\section{Metodologia}

Inicialmente, levantaram-se os títulos de todos dos trabalhos apresentados nos quatro eventos mencionados, e, por meio da ferramenta de busca por palavra disponível no software Word, buscou-se a palavra qualidade nos títulos. Essa busca indicou 20 artigos, que foram impressos, e cuja distribuição temporal foi a seguinte: 1) dois no evento de 2004: Rodrigo, Pereira e Siqueira (2004); Prado e Santos (2004); 2) cinco no evento de 2006: Guarita e Urdan (2006); Marchetti, Prado e Cuperschmid (2006); Baptista e Mazzon (2006); Durão et al. (2006); Xavier et al. (2006); 3) sete no evento de 2008: Koetz, Santos e Kopschina (2008); Torres (2008); Basso et al. (2008); Lopes, Alves e Leite (2008); Lopes, Alves e Leite (2008); Rodrigues (2008); Cavalcanti (2008); 4) seis no evento de 2010: Perez e Rodrigues (2010); Koetz e Costa (2010); Barbosa et al. (2010); Hernandez et al. (2010); Tinoco, Pereira e Ribeiro (2010); Cerchiaro e Mota (2010). Em seguida, identificaram-se os enfoques utilizados pelos autores em seus referenciais teóricos. Para tanto, utilizou-se o recurso de observar os subtítulos de seus respectivos referenciais teóricos, onde normalmente estão indicados terceiros construtos que compõem a linha de raciocínio científico relativo à avaliação - ou à mensuração - da qualidade percebida de um produto ou de um serviço.

Vale salientar, aqui, duas particularidades nesta análise: 1) excluiu-se a consideração à palavra qualidade eventualmente mencionada - isoladamente ou em conjunto com outra palavra - nos subtópicos, 
partindo-se do princípio de que o assunto estaria implícito no referencial teórico de todos os artigos; 2) excluíram-se subtópicos que seriam específicos a instrumentos utilizados para a mensuração da qualidade percebida, tais como o modelo Servqual - conforme encontrado em Cerchiaro e Mota (2010) - e a escala Retail Service Quality, conforme encontrado em Lopes, Alves e Leite (2008). Quando o artigo analisado não apresentava esse sistema de utilização de subtítulos com a indicação dos tópicos abordados no referencial teórico - como ocorre em Torres (2008) -, buscaramse indicadores internos ao material que esclarecessem seu embasamento teórico; a tática para tanto foi o exame do seu conteúdo por meio da leitura, buscando-se indicações do(s) próprio(s) autor(es) sobre qual teria sido aquele embasamento.

Em seguida à identificação dos tópicos, foram selecionados aqueles abordados por pelo menos dois artigos da amostra total. Em cada um deles foi levantada a literatura citada, com a indicação dos nomes dos autores mencionados e uma breve menção de sua linha de raciocínio resgatada pelo(s) autor(es) dos 20 artigos.

Vale notar que, como forma de se organizar a análise dos trabalhos selecionados, foi verificado se havia, nos respectivos resumos dos 20 artigos da amostra, a menção a algum outro componente de Marketing além da qualidade, que também teria sido utilizado na investigação. Verificou-se que, à exceção de três artigos, os demais lançaram mão desse recurso, tendo sido localizados 13 desses componentes (ver Quadro 1). Os mais utilizados foram satisfação e lealdade/confiança/comprometimento, em respectivamente quatro e três artigos. Logo em seguida na preferência, com dois artigos utilizando-os, destacaram-se relacionamento, valor, marca, dramaturgia e propaganda. Com menção em somente um artigo situaram-se preço, encontros, imagem, gerenciamento de impressões, emoções, ambiente e marca.

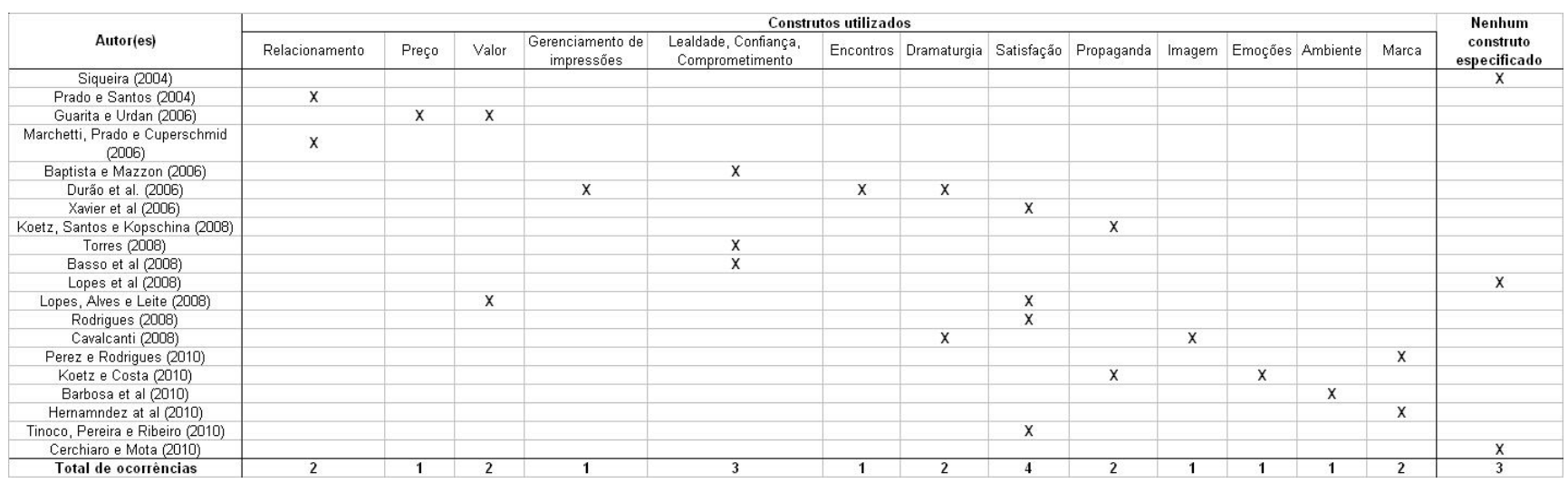

Quadro 1 - Construtos utilizados nos referenciais teóricos dos 20 artigos com a palavra qualidade no título apresentados nos EMAs de 2004 a 2010.

Fonte: Elaboração própria, com base nos EMAs.

Após esse esforço, teceram-se considerações finais sobre o material avaliado, e foram lançadas propostas de futuros estudos.

\section{RESULTADOS DA REVISÃo DA LITERATURA}

Revisão dos artigos que utilizaram a satisfação Xavier et al. (2006), Lopes, Alves e Leite (2008),
Rodrigues (2008) e Tinoco, Pereira e Ribeiro (2010) foram os autores que utilizaram a satisfação para complementar suas pesquisas sobre qualidade. $\mathrm{O}$ foco de Xavier et al. (2006) situou-se no relacionamento direto entre satisfação e qualidade, tendo os autores partido do princípio de que, a partir das expectativas criadas a respeito de uma determinada oferta de bens 
ou serviços, pode-se considerar que a satisfação traduz o grau de congruência entre as expectativas e as consequências da compra. Inicialmente eles se basearam em Karsaklian (2000), em Boulding et al. (1993) e em Johnson, Anderson e Fornell (1995), tendo relembrado que a satisfação pode ser vista enquanto avaliação de uma experiência particular com certo produto ou serviço, e enquanto a experiência total do consumo de um produto ou uso de um serviço de forma cumulativa. Em seguida, eles remeteram a Van Raaij (1981) e a Wärneryd (1988), ao mencionarem que o conceito de satisfação com base na experiência acumulada é, sob o ponto de vista da psicologia, equivalente à noção subjetiva de bem-estar, representando o desempenho atual e no longo prazo. E, mais adiante, lançaram mão de Bolton e Drew (1991) e de Parasuraman, Zeithaml e Berry (1988) para passar à questão de a qualidade de um serviço ser descrita como uma forma de atitude, relacionada mas não equivalente - à satisfação, vez que esta resulta da comparação entre expectativa e desempenho. Finalmente, concluindo a discussão a respeito da satisfação, Xavier et al. (2006) colocam que haveria uma tentativa dos pesquisadores, como ocorre nos estudos empíricos de Yu e Dean (2001), de verificar se sua gênese partiria de fatores afetivos ou cognitivos.

$\mathrm{O}$ segundo artigo que também trabalhou a satisfação foi o de Lopes, Alves e Leite (2008). De forma sucinta, eles inicialmente utilizamLáran (2003), que definiu a satisfação em dois grupos - como resultado de um processo e como uma parte de um processo -, com os aspectos cognitivos e afetivos dos julgamentos de satisfação sendo ressaltados no processo. Em seguida, mencionam Yi (1990) e Semenik e Bamossy (1996) para quem a satisfação é um julgamento que o consumidor faz depois de um encontro como fornecedor de serviços ou de produtos.

O referencial teórico do artigo de Rodrigues (2008) inicialmente cita Rossi e Braga (2004), salientando a necessidade de se interrogar diretamente o cliente e de se medir formalmente o seu grau de (in)satisfação, na busca pela convergência entre qualidade oferecida pelo produtor e percebida pelo cliente. Mencionando
Albrecht (1998) e Kotler (2000), a autora frisa que as empresas que se sobressaem são aquelas que conhecem as expectativas e as necessidades de seus clientes. Ela se preocupa também em buscar, na literatura, autores que diferenciaram qualidade de satisfação; isso foi encontrado em Machado (2004), para quem satisfação seria um julgamento de qualidade ao longo do tempo, e em Anderson, Fornell e Lehman (1994), para quem a avaliação da satisfação depende de uma experiência com o serviço - diferentemente do que ocorre com a percepção de qualidade -, assim como do valor e da percepção do consumidor no momento do consumo.

Finalmente, o último artigo a tratar da satisfação em seu referencial teórico foi o de Tinoco, Pereira e Ribeiro (2010). Inicialmente referindo-se a Oliver $(1980,1993)$, os autores lembram que ele foi um dos primeiros autores a propor um modelo de satisfação do cliente baseado no paradigma da desconfirmação das expectativas. Em seguida, eles se preocupam em ressalvar que Churchill e Surprenant (1982), Bearden e Teel (1983) e Tsiros, Mittal e Ross. (2004) avaliaram e confirmaram esse modelo, assim como salientam que outros determinantes foram estudados e incluídos nos modelos de satisfação dos clientes por parte de Spreng, Mackenzie e Olshavsky (1996), de Olshavsky e Kumar (2001), de Andreassen e Lindestad (1998), de Bei e Chiao (2001) e de Liang e Zhang (2009). A pesquisa a respeito da determinação da satisfação dos clientes, principalmente pela qualidade percebida, tambémé objeto da revisão da literatura nessa pesquisa - com base em Choi et al. (2004) e em Jiang e Wang (2006) -, assim como pelo valor, com base em Whittaker, Ledden e Kalafatis (2007) e em Vlachos e Vrechopoulos (2008). Os autores aproveitam para lembrar um trabalho anteriormente realizado por dois deles (TINOCO; RIBEIRO, 2007), em que é proposta uma abordagem para a modelagem da satisfação de clientes de serviços a partir da identificação das relações entre alguns destes múltiplos determinantes encontrados na literatura: imagem corporativa, desconfirmação de expectativas, emoções vivenciadas, desejos pessoais, expectativas, qualidade percebida, 
valor percebido, satisfação e preço do serviço.

\section{Revisão dos artigos que utilizaram lealdade e ou confiança e ou comprometimento}

Baptista e Mazzon (2006), Basso et al. (2008) e Torres (2008) foram os autores que utilizaram lealdade e ou confiança e ou comprometimento para complementar suas pesquisas sobre qualidade. Baptista e Mazzon (2006) iniciam sua revisão bibliográfica apontando Rosenberg e Czepiel (1983) e Andreassen e Lindestad (1998) enquanto autores que se preocuparam em mostrar a necessidade de se perseguir a retenção dos clientes, conceito intimamente relacionado à lealdade do consumidor. Já este último é por eles apresentado como elemento complexo há muito estudado sob as abordagens comportamental e atitudinal, tal como fizeram Mowen (2003), Assael (1998), Seth el al. (2001), Neal (1999), Lawrance (1969), Tucker (1964), Blattberg e Sem (1976), e Wind e Frank (1967). Em seguida eles passam à discussão sobre a importância de se distinguir a lealdade verdadeira da lealdade espúria, recorrendo a Bennett e Rundle-Thiele (2002) e a Day (1969). Mais à frente, relembram o papel dos processos cognitivos e afetivos na geração de preferências com base em Oliver (1999) e em Jacoby e Kyner (1973), chegando em seguida ao estudo de Dick e Basu (1994), que parte de uma abordagem composta em que está proposta uma estrutura conceitual para compreender melhor a dinâmica da lealdade dos consumidores.

O estudo da lealdade do cliente leva Baptista e Mazzon (2006) a abordarem também Seth e Park (1974), cuja pesquisa destacou os aspectos cognitivos, afetivos e conativos da atitude enquanto determinantes da lealdade, composta de três dimensões distintas que, se combinadas, podem resultar em sete diferentes tipos de lealdade à marca. Eles lembram que esse enfoque misto de comportamento de recompra com atitude também foi utilizado por Baldinger e Rubinson (1996), cuja proposição de outra tipologia contribuiu academicamente ao trazer evidências empíricas de que a lealdade atitudinal contribui tanto para a manutenção como para a geração da lealdade comportamental. Já completando sua revisão bibliográfica, Baptista e Mazzon (2006) abordam o fato de que realizar novas transações com a empresa é aspecto muito importante (ou mesmo indissociável) da existência de lealdade, pois se encontra no cerne da própria definição que diversos autores apresentam para a lealdade. Assim, o questionamento direto ao cliente dessa intenção de recompra se configura em indicador usual para mensuração da lealdade, ainda que se caracterize como um estimador possivelmente enviesado da probabilidade efetiva de recompra, conforme pesquisaram diversos autores: Prado (2004), Gonçalves Filho et al. (2003), Lau e Lee (2002), Bloemer e Odekerken-Schröder (2002), Santos e Rossi (2002), Sirdeshmukh, Singh e Sabol (2002), Srinivasa, Anderson e Ponnavolu (2002), Bei e Chiao (2001), Bloemer, Chaudhuri e Holbrook (2001), Mathwick, Malhotra e Rigdon (2001), Urdan e Zuniga (2001), Cronin, Brady e Hult (2000), Pritchard, Havits e Howard (1999), Sirohi, McLaughlin e Wittink (1998), Taylor e Baker (1994), e Zeithaml, Berry e Parasuraman (1996).

Finalmente, ao encerramento, Baptista e Mazzon (2006) remetem a autores que vinculam a lealdade a uma predisposição para comunicação boca a boca positiva por parte do cliente: Cortimiglia et al. (2003); Nijssen et al. (2003); Santos e Rossi (2002); Verhoef, Franses e Hoekstra (2002); Cronin, Brady e Hult (2000) e Yu e Dean (2001).

O segundo artigo selecionado que constrói seu referencial teórico ancorado na lealdade é o de Basso et al. (2008), que ampliam sua pesquisa incorporando mais dois elementos afins a este: confiança e comprometimento. Dentre os autores utilizados no que diz respeito à lealdade se destacam, além de clássicos como Oliver (1999), Newman e Werbel (1973), Day (1970) e Dick e Basu (1994), obras mais recentes. Dentre estas, um dos destaques é a de Laran e Espinoza (2004), que confirmaram empiricamente que o índice de recompra nem sempre indica lealdade à empresa: a compra pode ter sido em função de outros fatores, como preço, conveniência ou até mesmo lealdade a mais de uma marca. Também é resgatado o trabalho 
de Sirdeshmukh, Singh e Sabol (2002), para quem confiança, percepção de valor e satisfação são consideradas antecedentes da lealdade. Mais adiante, Basso et al. (2008) referem-se ao trabalho de Gounaris e Stathakopoulos (2004), que classificaram quatro tipos de lealdade - a não lealdade, a cobiça à lealdade, a lealdade inercial e a lealdade premium - e ao de Hennig-Thurau e Klee (1997), para quem a qualidade, a confiança e o comprometimento se relacionam entre si e são capazes de, direta ou indiretamente, influenciar a lealdade do consumidor.

Passando ao estudo da confiança, Basso et al. (2008) recorrem a Perin et al. (2004), a Moorman, Zaltman e Deshpandé (1992), a Morgan e Hunt (1994), a Gabarino e Johnson (1999) e a Masterson et al. (2000) para lembrar que ele passou a desempenhar fundamental importância para as organizações devido à maior percepção de risco e incerteza para os clientes. E que, principalmente sob a ótica dos serviços, a confiança é tratada como um antecedente da lealdade, por representar a integridade e a dignidade percebidas por um parceiro no outro, que a faça ser vista como umingrediente essencial para o sucesso dos relacionamentos, segundo Berry (1995), Dwyer, Schurr e Oh (1987), Moorman, Deshpandé e Zaltman (1993) e Morgan e Hunt (1994). Em seguida, Basso et al. (2008) lembram, com base nos trabalhos de Moorman, Zaltman e Deshpandé (1993) e de Singh e Sirdeshmukh (2000), que a confiança é vista geralmente sob dois aspectos: como crença, sentimento ou expectativa, e como comportamento. Também pode também ser encarada como um estado psicológico e como uma escolha de comportamento, de acordo com Kramer (2000). Já pelo lado dos clientes de serviços, em que existe forte confiança no atributo confiabilidade, eles frisam que os benefícios trazidos pela confiança são importantes, conforme apontou a pesquisa de Bejou, Ennew e Palmer (1998), estando o desenvolvimento da confiança intimamente relacionado à duração do tempo do relacionamento, segundo Swan e Nolan (1985). Na esteira desse raciocínio se situam os trabalhos de Lagace, Dahlstrom e Gassenheimer (1991), Morgan e Hunt (1994) e
Oakes (1990), que apontam que a confiança é vista como de considerável importância no processo de construção e de manutenção de relacionamentos.

Finalmente, no que tange ao comprometimento, Basso et al. (2008) recorrem a Garbarino e Johnson (1999), a Sheth e Parvatiyar (1995) e a Morgan e Hunt (1994) para expor que ele é considerado central para o estudo do comportamento relacional com o consumidor, sendo capaz de influenciar a lealdade e sendo considerado variável-chave em modelos de relacionamento. Por outro lado, eles também lembram que a especificação da variável comprometimento ainda não está clara na literatura, conforme Prado e Santos (2003), porque alguns autores preferem uma visão independente, tais como Hennig-Thurau e Klee (1997), enquanto outros são partidários da vinculação entre comprometimento e lealdade, tais como Chaudhuri e Holbrook (2001).

Um outro aspecto resgatado no referencial teórico de Basso et al. (2008) diz respeito às consequências do comprometimento dentro das organizações. Conforme Morgan e Hunt (1994), essas consequências incluem o decréscimo de turnover, maior motivação e aumento do comportamento cooperativo. Em paralelo, eles lembram que o comprometimento entre um consumidor e uma organização pode afetar a lealdade, de modo a criar um sentimento de obrigação entre os atores, de acordo com Vieira e Damacena (2007), e que diversos estudos têm encontrado relações entre o comprometimento e a lealdade, tais como em Grönroos (1994) e em Pritchard, Havitz e Howard (1999).

O terceiro e último artigo que trabalha confiança é o de Torres (2008). Contudo, embora sua proposta seja a de testar um modelo que avalie as relações entre qualidade percebida pelo cliente do varejo virtual e comprometimento, lealdade e confiança, tal esforço é feito semuma revisão bibliográfica específica a respeito desses últimos.

\section{Revisão dos artigos que utilizaram relacionamento}

Prado e Santos (2004) e Marchetti, Prado e 
Cuperschmid (2006) foram os autores que utilizaram o relacionamento para complementar suas pesquisas sobre qualidade. Embora o propósito de Prado e Santos (2004) seja, por meio da proposta de modelo estrutural, enfocar a avaliação da relação entre empresas e consumidores finais, e os resultados do processo de relacionamento, os autores o fazem sem lançar mão de revisão bibliográfica específica a respeito.

O segundo artigo localizado foi o de Marchetti, Prado e Cuperschmid (2006), cujo propósito é apresentar um modelo para avaliação da qualidade do relacionamento em companhias distribuidoras de gás. Como no caso de Prado e Santos (2004), eles o fazem sem lançar mão de revisão bibliográfica específica a respeito do relacionamento.

\section{Revisão dos artigos que utilizaram valor}

Guarita e Urdan (2006) e Lopes, Alves e Leite (2008) foram os autores que utilizaram o valor para complementar suas pesquisas sobre qualidade. Além de lançarem mão do construto valor, Guarita e Urdan (2006) também trabalharam o preço. Inicialmente eles se preocupam em deixar claro que valor é ainda tratado de forma ambígua na literatura acadêmica, conforme pode definir tanto aquilo que o cliente recebe do fornecedor quanto aquilo que o fornecedor recebe do cliente, conforme Woodwall (2003), podendo ser usado de diferentes maneiras, dependendo daquilo que mais se valoriza, de acordo com Zeithaml (1988), Holbrook e Corfman (1985) e Gremaud et al. (2003). Em seguida, os autores lembram a dificuldade de se conceituar e de se medir o valor percebido, de acordo com Parasuraman, Zeithaml e Berry (1985, 1988), Garvin (1992) e Gale (1996). Finalmente, eles chegam à discussão sobre a relação entre qualidade e valor, para tanto resgatando as contribuições de Dodds, Monroe e Grewal (1991) e de Teas e Agarwal (2000). Depois, eles tocam em outro ponto muito comum na literatura de Marketing atinente a valor: a diferença entre qualidade percebida e valor percebido. No caso, eles lançam mão dos trabalhos de Monroe e Krishnan (1985) e de Gronroos (2001).

O segundo trabalho que enfoca valor é o de Lopes,
Alves e Leite (2008). Em sua curta revisão teórica a respeito, à semelhança de Guarita e Urdan (2006), os autores resgatam os pontos de vista Woodall (2003), de Zeithaml (1988), e de Holbrook e Corfman (1985). E, em seguida, eles utilizam Sirdeshmuck, Singh e Sabol (2002) para mostrar a variedade de definições a respeito.

\section{Revisão dos artigos que utilizaram dramaturgia}

Durão et al. (2006) e Cavalcanti (2008) foram os autores que utilizaram a dramaturgia para complementar suas pesquisas sobre qualidade. $\mathrm{O}$ artigo de Durão et al. (2006) estuda encontros de serviços e as teorias dos papéis e dos roteiros. Ao rever trabalhos acadêmicos sobre a teoria dos papéis, os autores enfocam Bitner, Booms e Tetreault (1990), que dizem que as partes envolvidas no processo de entrega de um serviço são consideradas responsáveis por papéis que interpretam durante a interação existente a cada encontro. Sua primeira preocupação é indicar que essa teoria deriva da Teoria da Penetração Social, conforme proposta por Altman e Taylos (1973), da abordagem da interação social, conforme proposta por Goffman (1975) e por Simmel (1977, 1990), e de elementos diádicos da teoria das trocas sociais (HOMANS, 1961; KELLEY; THIBAUT, 1993), concentrando seu foco na interatividade dentro de padrões de trocas sociais. Ademais, Durão et al. (2008) deixam claro, apoiados em Biddle (1986), que essa teoria é considerada um movimento - e não uma escola teórica única - que explica os papéis ao considerar que pessoas são membros de posições sociais e que carregam consigo expectativas sobre os próprios comportamentos e daqueles com quem interagem. Eles avançam no assunto, explicando que, das cinco perspectivas da teoria dos papéis apontadas por Biddle (1986) como existentes na literatura funcional, interacionista simbólica, estrutural, organizacional e cognitiva-, existem dois enfoques mais divergentes, de acordo com Stryker e Staham (1985): um de ênfase estruturalista e outro comênfase interacionista.

O segundo artigo a utilizar a dramaturgia em seu 
referencial teórico é o de Cavalcanti (2008). Nele, a autora resgata Grove e Fisk (1989), para quem a dramaturgia é baseada no comportamento como uma metáfora dramática e na representação das interações sociais como teatrais. No caso, as falas de um ator numa peça seriam representativas de um desempenho de um papel que é a fonte da motivação do personagem, sendo que essas motivações podem ser identificadas como sendo as normas sociais e os objetivos pessoais que limitam os comportamentos individuais no dia-a-dia, conforme Grayson e Shulman (1999). E cada performance dos atores teria uma intenção de criar uma variedade de reações e respostas na audiência, de acordo com Baron, Harris e Harris (2001).

Revendo o conceito de interações sociais a partir de Babcock (1989), Cavalcanti (2008) explica que se trata de esforço cooperativo de apresentar um desempenho aceitável e convincente, no qual o indivíduo (ou ator) irá promover uma imagem particular e implicitamente pedir a outros que acreditem no personagem. Nesse sentido, o ator não está sozinho, ele está sempre trabalhando em conjunto com a audiência. E a autora prossegue, fazendo um paralelo entre a dramaturgia e os encontros de serviços - com base em John (1996), em Grove e Fisk (1983), em Grove, Fisk e Dorsh (1998) e em Goodwin (1996) , indicando a importância do paralelo entre atuações teatrais e desempenhos organizacionais trazido pelo trabalho de Schreyögg e Höpfl (2004).

\section{Revisão dos artigos que utilizaram propaganda}

Koetz, Santos e Kopschina (2008) e Koetz e Costa (2010) foram os autores que utilizaram a propaganda para complementar suas pesquisas sobre qualidade. Koetz, Santos e Kopschina (2008) inicialmente alertam que a forma de abordagem do tema nos estudos de comportamento do consumidor segue linhas de trabalho distintas, com destaque para as de Chandy et al. (2001) e de Vakratsas e Ambler (1999). Em seguida, os autores baseiam-se em Kirmani e Rao (2000) para chamar a atenção de que a qualidade dos produtos das empresas que investem grandes montantes em propaganda é vista pelos consumidores como sendo boa, muito embora haja estudos - tais como o de Kirmani (1997) e o de Barone, Taylor e Urbany (2005) - apontando que existem diversos fatores que atuam nesse mecanismo.

O segundo trabalho da amostra cujo referencial teórico aborda a propaganda é o de Koetz e Costa (2010), mas estudando-a enquanto veículo de sinalização da qualidade. As autoras indicam, inicialmente, que Nelson (1974) foi um dos precursores a analisar a relação entre o volume investido em propaganda e a informação sobre o produtor ao público consumidor. Em seguida, elas encerram sua pesquisa bibliográfica resgatando os trabalhos de Kirmani e Wright (1989) e de Kirmani (1990), que analisaram a influência dos investimentos publicitários na percepção de qualidade dos consumidores.

\section{Revisão dos artigos que utilizaram marca}

Perez e Rodrigues (2010) e Hernandez et al. (2010) foram os autores que utilizaram a marca para complementar suas pesquisas sobre qualidade. Perez e Rodrigues (2010) inicialmente mencionam Perez $(2004,2007)$ e sua definição de que as marcas são o elo simbólico de ligação entre as organizações e as pessoas, com essa construção de identidade começando com a própria escolha do nome da marca, e passando pelas diferentes expressividades mencionadas por Gobé (2003), que a organização constrói no tempo. Ao final de sua pesquisa bibliográfica, eles chamam a atenção para os estudos de gestão de marcas realizados por Aaker (1991), por Keller (1997) e por Kapferer (1993), além de resgatar a pesquisa sobre marcas de luxo realizada por Thomas (2008). Mais à frente eles salientam que há dois movimentos bastante evidentes em relação às marcas, conforme apresentado por McCraken (2003): 1) em direção à diferenciação social, as marcas são o símbolo do sucesso econômico, do êxito, do status elevado; e 2) em direção à afirmação da identidade, da autenticidade, da personalização e, muitas vezes, até da negação à moda.

O segundo artigo da amostra estudada que salienta 
marca como suporte em sua pesquisa sobre qualidade é o de Hernandez et al. (2010). No caso, seu foco é a extensão de marcas, com foco específico nas marcas corporativas. Ao explicitarem-no, os autores lançam mão do estudo de Grime, Diamantopoulos e Smith (2002), que indicou que extensões de marcas promovem lembrança imediata, proporcionando uma forma relativamente fácil e barata para se entrar em novos mercados. Também aproveitam para relembrar as pesquisas de Smith e Park (1992), de Balachander e Ghose (2003) e de Swaminathan, Fox e Reddy (2001) para afirmar que, além de facilitar a aceitação do novo produto, a utilização de uma marca conhecida para o lançamento de produtos e serviços também tem efeitos benéficos para a marca-mãe: maior eficiência dos investimentos em propaganda, melhoria da imagem da marca e o aumento da visibilidade da marca-mãe. Em seguida, os autores lançam seu foco ao estudo da extensão de marcas corporativas de serviços. Com base em Iacobucci (1992), em Iacobucci e Ostrom (1996), em Rao, Qu e Ruekert (1999), e em Keller e Aaker (1998), eles indicam ser este estudo especialmente atraente, por dois motivos: 1) dada a complexidade e intangibilidade dos serviços, a aquisição de serviços é percebida como mais arriscada que a de um produto tangível, e 2) porque a utilização de uma imagem corporativa bem estabelecida pode aumentar a credibilidade e facilitar a percepção de qualidade do novo serviço. Eles também aproveitam para salientar que tanto Völckner e Sattler (2006) quanto Bottomley e Holden (2001) já indicaram que os principais determinantes para a aceitação de uma extensão de marca são a similaridade percebida entre a marca e o novo produto e a qualidade da marcamãe. Entretanto, ressaltam que, até à época de seu levantamento, os estudos anteriores sobre extensões de marcas de serviços (RUYTER; WETZELS, 2000; RIEL; LEMMINK; OUWERSLOOT, 2001; LEI; PRUPPERS; OUWERSLOOT, 2004; PINA et al., 2006) se haviam abstido de examinar experimentalmente a extensão de marcas corporativas de serviços para outras categorias de serviços. Contudo, Hernadez et al. (2010) salientam que, de acordo com Ruyter e Wetzels (2000), a despeito da importância do setor de serviços, e de o citado trabalho de Riel, Lemmink e Ouwersloot (2001) indicar claramente que há diferenças na avaliação de extensões de marcas entre produtos tangíveis e intangíveis, a maior parte dos estudos tem se enfocado no estudo de produtos tangíveis e em marcas individuais. E aproveitam para indicar que uma das exceções é o citado trabalho de Pina et al. (2006).

\section{CONSIDERAÇÕES FINAIS, LIMITAÇÕES E SUGESTÕES PARA FUTUROS ESTUDOS}

A proposta de um artigo de revisão bibliográfica, como o nome diz, é mostrar quais os autores e as respectivas linhas de raciocínio foram utilizados emuma amostra de textos, selecionada a partir de um critério em especial. Adicionalmente, sua virtude pode ser expandida a partir de uma avaliação crítica daquilo que foi encontrado.

Passando-se primeiramente à descrição dos dados macro que o levantamento indicou, tem-se, como ponto inicial mais relevante, o fato de que os quatro EMAs mapeados apresentaram, em seu conjunto, 425 artigos aprovados, e, destes, tão somente 20 trouxeram o construto qualidade em seus títulos - ou seja, menos de $5 \%$. Embora seja provável que outros artigos veiculados nos eventos possam ter envolvido esse elemento, embora sem trazê-lo no título, é legítimo supor-se que poucos o teriam feito sem uma menção no título. Ou seja, pelo menos no âmbito dos EMAs, o interesse acadêmico pelos estudos versando sobre qualidade não parece elevado. A confirmação - ou não - dessa situação poderia ser obtida em futuros estudos que mapeassem os construtos de todos os 425 artigos, de forma a verificar quais os mais assíduos, e comparando-os com a frequência apurada aqui.

A segunda constatação é que muito poucos artigos se atêm a explorar o construto qualidade de forma isolada, sem lançar mão de outro(s) que ajude(m) na construção dos raciocínios. E, dentre esses construtos auxiliares, o mais utilizado é a satisfação. Esta preferência faz sentido, tendo em vista ambos os construtos - satisfação e qualidade - serem 
praticamente complementares na literatura de Marketing - e, muitas vezes, até confundidos. Conforme Zeithaml, Bitner e Gremler (2011), em serviços a satisfação do cliente advém de quatro fatores: fatores situacionais, preço do serviço, fatores pessoais, e da percepção do cliente de que o serviço tem qualidade, sendo esta última entendida como atitude de longo prazo, que reflete a avaliação de percepções de serviços realizados em pontos específicos do tempo.

Dentre os demais elementos utilizados subsidiariamente nas pesquisas, os mais destacados foram a lealdade, a confiança e o comprometimento. Utilizados nesta pesquisa como um único bloco, são tradicionalmente vistos, nas pesquisas de Marketing sobre qualidade, como consequências naturais da satisfação que a ela se segue - assim como seus antônimos seriam consequências naturais da insatisfação - num encadeamento lógico: se percebo qualidade, posso ficar satisfeito e, por consequência, me tornar leal à marca ou ao prestador do serviço, nela(e) confiando e com ela(e) sentindo-me comprometido. Ou seja, como já dizia Oliver (1999), a satisfação é um caminho indispensável para o desenvolvimento da lealdade.

A terceira constatação é a de que poucos artigos lançaram mão, simultaneamente, de mais do que apenas um elemento subsidiário à qualidade: um artigo utilizou três outros e três artigos utilizaram dois outros. Isso pode indicar tão simplesmente que os autores preferiram um comportamento mais comedido, visando não só facilitar-lhes a consecução como também diminuir a probabilidade de questionamentos metodológicos que colocassem em risco a validade dos resultados das pesquisas e, por tabela, a aprovação do material no evento.

Uma quarta e última constatação remete aos elementos que foram menos utilizados subsidiariamente nesses estudos sobre qualidade: preço, gerenciamento de impressões, encontros, imagem, emoções e ambiente. Essa parcimônia pode sugerir que se trata de domínios entendidos como pouco interessantes à academia e ou como mais difíceis de serem trabalhados.
Uma sugestão para dirimir esta dúvida seria a realização de pesquisa semelhante em eventos internacionais de Marketing de primeira linha, de forma a se avaliar se haveria similaridade nessa tendência.

Passando-se às conclusões a respeito das referências em si utilizadas pelos diversos autores, verifica-se que, de uma forma geral, estes tenderam à diversidade: dentro de cada conjunto de trabalhos que lançaram mão de um mesmo elemento para suportar a pesquisa sobre qualidade, raramente se observou uma similaridade no referencial. Ao contrário, com exceção obviamente de trabalhos seminais, o que se vê é uma tendência à pluralidade.

Esse fenômeno trouxe, a reboque, enfoques diferenciados ao referencial teórico e às linhas de raciocínio de cada um dos trabalhos. Eé relevante notar que isso ocorreu mesmo naqueles casos em que o foco da pesquisa remete à discussão acadêmica exaustivamente presente na literatura. Foi o caso dos quatro trabalhos que utilizaram o construto satisfação: este e o construto qualidade, como se viu anteriormente, têm sido muito frequentemente analisados em uníssono na área de Marketing, o que poderia sugerir certa tendência à congruência ao se montar os referenciais teóricos. Contudo, os artigos de Xavier et al. (2006), de Lopes, Alves e Leite (2008), de Rodrigues (2008) e de Tinoco, Pereira e Ribeiro (2010) primam pela diversidade dos autores que embasaram seus respectivos referenciais - o que sinaliza esforço bemsucedido, por parte dos pesquisadores selecionados nos EMAs, ao embasar seus estudos em fontes diferenciadas, de forma a reforçar-lhes a originalidade.

É importante ressaltar que este artigo, assim como qualquer outro texto científico, possui limitações, sendo uma delas o tamanho limitado da amostra. Outra limitação remete à precisão e ao rigor metodológico do método de seleção de corpus com base em indexação de palavras. Este pode tão somente indicar relacionamento conceitual circunstancial entre os elementos identificados, assim como não está garantida a clareza quanto à relação entre eles.

Finalmente, como mais uma sugestão de futuros estudos, indica-se a execução de pesquisa semelhante 
a esta no âmbito dos encontros da Associação Nacional de Pós-Graduação em Administração, em seguida comparando-se os resultados.

\section{REFERÊNCIAS}

AAKER, D. A. Managing brand equity: capitalizing on the value of a brand name. New York: The Free Press, 1991.

ALBRECHT, K. A única coisa que importa: trazendo o poder do cliente para dentro da empresa. São Paulo: Pioneira, 1998.

ALTMAN, I.;TAYLOR, D. Social Penetration: The Development of Interpersonal Relationships. NewYork: Holt, Rinehart and Winston, 1973.

ANDERSON, E.; FORNELL, C.; LEHMANN, D. Customer Satisfaction, Market Share, and Profitability: Findings From Sweden. Journal of Marketing, vol. 58, Jul., 1994.

ANDREASSEN, T.; LINDESTAD, B. Customer loyalty and complex services. International Journal of Service Industry Management, v. 9, n. 1, p. 723, 1998.

ASSAEL, $\mathrm{H}$. Consumer Behavior and marketing action. Cincinnati: South-Western College Publishing, 1998.

BABCOCK, M. K. The dramaturgic perspective: Implications for the study of person perception. European Journal of Social Psychology, v.19, n. 4, 1989.

BALACHANDER, S.; GHOSE, S. Reciprocal spillover effects: Astrategic benefit of brand extensions. Journal of Marketing, v. 67, p. 4-13, 2003.

BALDINGER, A.; RUBINSON, J. Brand loyalty: the link between attitude and behavior. Journal of Advertising Research. v. 36, p. 22-34, nov., 1996.

BAPTISTA, P. P.; MAZZON, J. A. Relação Entre Qualidade Percebida e Lealdade do Consumidor: Um Estudo Aplicado ao Setor Varejista na Internet. ENCONTRO DE MARKETING DA ANPAD, 2., 2006, Rio de Janeiro, Anais..., Rio de Janeiro: EMA, 2006.

BARBOZA, M. V.; CARNEIRO, J. V.C.; ARRUDA, D. M. O.; MOURA, H. J. Impacto do Aroma sobre a Percepção da Qualidade no Varejo: experimento em ambiente real de loja. ENCONTRO DE MARKETING DAANPAD.,4.,2010. Florianópolis. Anais... Florianópolis: EMA, 2010.

BARON, S.; HARRIS, K.; HARRIS, Richard. Retail Theater: the "intended effect" of the performance.

Journal of Service Research, v.4, n.2, 2001

BARONE, M., TAYLOR, V., URBANY, J. Advertising Signaling Effects for New Brands: The Moderating Role of Perceived Brand Differences. Journal of Marketing Theory and Practice, v. 13, n.1, p. 1-13, 2005.

BASSO, K; SCHWAB, A.; PÓlVORA, R. P.; MARQUES, L. T.; PEREIRA, M. C.; SLONGO, L. A. Relações de Interdependência entre Confiança, Comprometimento, Qualidade Percebida e Lealdade em Estudantes do Ensino Superior. ENCONTRO DE MARKETING DA ANPAD., Curitiba., 3., 2008. Anais... Curitiba: EMA, 2008.

BEARDEN, W.; TEEL, J. Selected Determinants of Consumer Satisfaction and Complaint Reports. Journal of Marketing Research, v. 20, p. 21-28, Feb., 1983.

BEI, L.; CHIAO, Y. An integrated model for the effects of perceived product, perceived service quality, and perceived price fairness on consumer satisfaction and 
loyalty. Journal of Consumer Satisfaction,. v.14, p. 126-140, 2011.

*BEJOU, D.; ENNEW, C. T.; PALMER, A. Trust, Ethics and Relationship Satisfaction. International Journal of Bank Marketing, v.16, n. 4, p. 170-175, 1998.

BENNETT, R.; RUNDLE-THIELE, S. A comparison of attitudinal loyalty measurement approaches. Journal of Brand Management, v. 9, n. 3, p.193-209, Jan., 2002.

BERRY, L. Relationship Marketing of Services: Growing Interest, Emerging Perspectives. Journal of Academy of Marketing Science, v. 23, p. 236-245, 1995.

BIDDLE, B.J. Recent Developments in Role Theory. Annual Review of Sociology, v.. 12, p. 67-92, 1986.

BITNER, M. Servicescapes: the impact of physical surroundings on customers and employees. Journal of Marketing, v. 56, p. 57-71, apr. 1992.

BITNER, M.J.; BOOMS, B.H.; TETREAULT, M.S. The Service Encounter: diagnosing favorable and unfavorable incidents. Journal of Marketing, v. 54, p. 71-84, Jan., 1990.

BLATTBERG, R.; SEM, S. Market segments and stochastic brand choice models. Journal of Marketing Research. v.13, p. 34-45, Feb., 1976.

BLOEMER, J.; ODEKERKEN-SCHRÖDER, G. Store satisfaction and store loyalty explained by customer and store-related factors. Journal of Consumer Satisfaction, Dissatisfaction And Complaining Behavior. v. 15, p. 68-80, 2002.

BOLTON, R. N.; Drew, J. H. A Multistage Model of Customers' Assesment of Service Quality and Value. Journal of Consumer Research v.54, p. 69-
82, abr. 1991.

BOULDING, W.; KALRA, A.; STAELIN,R.; ZEITHAML, V. Adynamic process model of service quality: from expectations to behavioral intentions. J. Market. Res. n. 30, p. 7-27, 1993.

BOTTOMLEY, P.A.; HOLDEN, S.J.S. Do we really know how consumers evaluate brand extensions? Empirical generalizations based on secondary analysis of eight studies. Journal of Marketing Research, v. 38, p. 494-500, 2001.

CAVALCANTI, G. G. Imagem e qualidade percebida: A perspectiva dramatúrgica do marketing de serviços. Curitiba. ENCONTRO DE MARKETING, 20083. Curitiba. Anais... Curitiba: EMA, 2008.

CHANDY, R. K., TELLIS, G. J., MACINNIS, D. J., THAIVANICH, P. What to Say When: Advertising Appeals in Evolving Markets. Journal of Marketing Research, v. 38, Nov. 2001.

CHAUDHURI, A.; HOLBROOK, M. B. The Chain of Effects from Brand Trust and Brand Affect to Brand Performance: The Role of Brand Loyalty. Journal of Marketing, v. 65, p.81-93, 2001

CERCHIARO, I. B.; MOTA, M. C. Avaliação da qualidade do serviço educacional numa IES particular. ENCONTRO DE MARKETING DA ANPAD, 4., 2010, Curitiba. Anais... Curitiba: EMA, 2010.

CHOI, K. S.; CHO, W. H.; LEE, S.; LEE, H.; KIM, $\mathrm{C}$. The relationships among quality, value, satisfaction and behavioral intention in health care provider choice: A South Korean study. Journal of Business Research, v. 57, p. 913-921, 2004.

CHURCHILL, G. A.; SURPRENANT, C. An investigation into the determinants of customer satisfaction. Journal of Marketing Research, v. 19, p. 491-504, nov.1982. 
Qualidade: uma revisão da literatura...

CORTIMIGLIA, M. N.; MACADAR, B. M.; DINATO, M. R. O impacto do tipo de relacionamento na lealdade do consumidor em um contexto de falhas de serviço: um estudo experimental no Brasil. ENCONTRO DA ANPAD, 27, Atibaia, 2003. Anais... Atibaia: ENANPAD 2003.

CRONIN, J.; BRADY, M.; HULT, G. Assessing the effects of quality, value and customer satisfaction on consumer behavioral intentions in service enviroments. Journal of Retailing. Summer, v. 76, p. 193-218, 2000

CRONIN, J.; TAYLOR, S. Measuring Service Quality: A Reexamination and Extension. Journal of Marketing. v. 56, p. 55-68, Jul., 1992

DAY, G. A two dimensional concept of brand loyalty. Journal of Advertising Research. v. 9, n. 3, p. 2935, Sep., 1969.

DAY, G. Buyer Attitudes and Brand Choice Behavior. New York: The Free Press, 1970.

DICK, A.; BASU, K. Customer loyalty: Toward an Integrated Conceptual Framework. Journal of the Academy of Marketing Science, v. 22, p. 99-113, 1994.

DODDS, W.B.; MONROE, K.; GREWAL, D. Effects of price, brand, and store information on buyer's product evaluations. Journal of Marketing Research, v.28, n.3, p. 307-319, 1991

DURÃO, A. F.; BARBOSA, M. L. A.; TEIXEIRA, A. K.; FARIAS, S. A. Gerenciamento de Impressões em Encontros de Serviços de Hospitalidade Contribui Para a Qualidade Percebida? ENCONTRO DE MARKETING DAANPAD, 2., 2006, Rio de Janeiro. Anais... Rio de Janeiro: EMA, 2006.

DWYER, R.; SCHURR, P.H.; OH, S. Developing Relationship Theory in Consumer Research. Journal of Marketing, p.11-27, 1987

GALE, B.T. Gerenciando o valor do cliente: criando qualidade e serviços que os clientes podem ver. São Paulo: Pioneira, 1996

GARBARINO, E.; JOHNSON, M. S. The Different Roles of Satisfaction, Trust, and Commitment in Customer Relationships. Journal of Marketing, v. 63, n. 2, 1999.

GARVIN, D. A. Gerenciando a qualidade: a visão estratégica e competitiva. Rio de Janeiro: Qualitymark, 1992

GOBÉ, M. A emoção das marcas. São Paulo: Negócio, 2003

GOFFMAN, E. A Representação do Eu na Vida Cotidiana. Rio de Janeiro: Vozes, 1975

GONÇALVES FILHO, C.; GONÇALVES, C.; SOARES, F; DO ESPÍRITO SANTO, N. Comércio eletrônico na Internet: uma pesquisa exploratória no mercado consumidor. ENCONTRO DAANPAD, 22, Anais..., 1998, Foz do Iguaçu: ENANPAD, 1998.

GOODWIN, C. Moving the Drama into the Factory: the Contribution of Metaphors to Service Research. European Journal of Marketing, v. 30, n. 9, 1996

GOUNARIS, S.; STATHAKOPOULOS, V. Antecedents and Consequences of Brand Loyalty: An Empirical Study. Journal of Brand Management, v. 11, n. 4, p. 283-306, 2004

GRAYSON, K.; SHULMAN, D. Impression Management in Services Marketing. In: SWARTZ, T. A.; IACOBUCCI, D. Handbook of services marketing e management. Thousand Oaks: Sage Publications, 1999.

GREMAUD, A.; MONTORO FILHO, A.; 
LANZANA, A.; LUQUE, C.; PINHO, C.; ALVES, D. Manual de economia. 4. ed. São Paulo: Saraiva, 2003

GRIME, I.; DIAMANTOPOULOS, A.; SMITH, G. Consumer evaluations of extensions and their effects on the core brand. Key issues and research propositions. European Journal of Marketing, v. 36, n. 11/12, p. 1415-1438, 2002

GRÖNROOS, C. The perceived service quality concept - a mistake? Managing Service Quality. v. 11 , n.3, p. 150-153, 2001

GRÖNROOS, C. Marketing: geranciamento e serviços. Rio de Janeiro: Elsevier, 2003.

GROVE, S.; FISK, R. Impression management in services marketing: a dramaturgical perspective. In: GIACALONE, Robert A; ROSENFELD, Paul (Eds.). Impression Management in the Organization. USA: LEA, 1989.

GROVE, S.; FISK, R.; DORSCH, M. Assessing the Theatrical Components of the Service Encounter: a Cluster Analysis Examination. The Service Industries Journal, v. 18, n. 3, 1998.

GUARIDA, C.; URDAN, A. Percepções de Qualidade, Sacrifício e Valor: uma investigação em serviços bancários. ENCONTRO DE MARKETING DAANPAD., 2. 2006, Rio de Janeiro. Anais... Rio de Janeiro: EMA, 2006.

HENNIG-THURAU, T.; GWINNER, K.; GREMLER, D. Understanding relationship marketing outcomes: an integration of relational benefits and relationship quality. Journal of Service Research. v. 4, p. 203-247, Feb. 2002

HENNIG-THURAU, T.; KLEE, A. The Impact of Customer Satisfaction and Relationship Quality on Customer Retention - A Critical Reassessment and
Model Development. Psychology \& Marketing, v. 14, p. 737-765, 1997

HERNANDEZ, J. M. C.; MENCK, T. M.; LOPEZ, D. M. G.; MOCELLIN, L. F. T. Extensão de Marcas Corporativas de Serviços: O Papel da Similaridade e da Qualidade Percebida da Marca.ENCONTRO DE MARKETING DA ANPAD, 2010, Florianópolis. Anais... Florianópolis; EMA, 2010.

HOLBROOK, M.; CORFMAN, K. Quality and value in the consumption experience: Phaedrus rides again. In: JACOBY, J.; OLSON, J. Perceived quality: how consumers view stores and merchandising. Lexington: Lexington Books, p. 31-51, 1985.

HOMANS, G. C. Social Behavior. New York: Harcourt Brace and World, 1961.

IACOBUCCI, D. An empirical examination of some basic tenets in services: goodsservices continua. In: SWARTZ, T.A.; BOWEN, D.E.; BROWN, S.W. (eds.), Advances in Services Marketing and management: Research and Practice, New York: JAI Press, 1992. v.1 p. 23-52.

IACOBUCCI, D.; OSTROM, A. Perceptions of services. Journal of Retailing and Consumer Services, v. 3, p. 195-212, 1996.

JACOBY, J.; KYNER, D. Brand loyalty vs. repeat purchasing behavior. Journal of Marketing Research. v.10, p. 1-9, Feb., 1973.

JIANG, Y.; WANG, C. The impact of affect on service quality and satisfaction: the moderation of service contexts. Journal of Services Marketing, v. 20, n. 4, p. 211-218, 2006.

JOHN, Joby. ADramaturgical View of the Health Care Service Encounter: cultural value-based impression management guidelines for medical professional behavior. European Journal of Marketing. v. 30, n.9, 1996. 
KAPFERER, J. Strategic brand management. New York: Free Press, 1993

KARSAKLIAN, E. Comportamento do Consumidor. São Paulo: Atlas, 2000.

KELLER, K. Strategic brand management. New York.: Prentice Hall, 1997.

KELLEY, H. ; THIBAUT, J. The social Psychology of Groups. New York: Wiley Third, Hamilton and Stewart, 1993.

KELLER, K.; AAKER, D. The impact of corporate marketing on a company's brand extensions. Corporate Reputation Review, v. 1, n. 4, p. 356378, 1998.

KIRMANI, A. The Effect of Perceived Advertising Costs on Brand Perceptions. Journal of Consumer Research, v. 17, p. 160-171, sep.1990.

KIRMANI, A. Advertising Repetition as a Signal of Quality: If it's Advertised so Often, Something Must Be Wrong. Journal of Advertising, v. 26, n.3, p. 7786, 1997.

KIRMANI, A.; RAO, A. No Pain, No Gain: ACritical Review of the Literature on Signaling Unobservable Product Quality. Journal of Marketing, n. 64 p. 6679, apr. 2000.

KIRMANI, A.; WRIGHT, P. Money Talks: Perceived Advertising Expense and Expected Product Quality. Journal of Consumer Research, n. 16 . 344-353, dec. 1989.

KOETZ, C.; COSTA, C. Penso, (Sinto) e Julgo - O Papel da Emoção na Avaliação de Novos Produtos em Processos de Sinalização da Qualidade por meio da Propaganda. ENCONTRO DE MARKETING DA ANPAD, 4., 2010, Florianópolis. Anais... Florianópolis: EMA, 2010.
KOETZ, C. ; SANTOS, C.; KOPSCHINA, L. A Sinalização da Qualidade por meio da Propaganda Proposições de Pesquisa, Implicações Gerenciais e Direções Futuras. ENCONTRO DE MARKETING DA ANPAD.3.,2008, Curitiba. Anais... Curitiba: EMA, 2008.

KOTLER, P. Administração de Marketing: a edição do novo milênio. 10.ed. São Paulo: Prentice Hall, 2000

KRAMER, R. Trust and Distrust in Organizations: Emerging Perspectives, Enduring Questions. Annual Review of Psychology, v. 50, p. 569-598, 2000.

LAGACE, R.; DAHLSTROM, R.; GASSENHEIMER, J. The Relevance of Ethical Salesperson Behavior on Relationship Quality: The Pharmaceutical Industry. The Journal of Personal Selling \& Sales Management. v. 11, n. 4; p. 3948, 1991.

LARAN, J. A influência da surpresa no processo emocional de formação da satisfação do consumidor. Dissertação (Mestrado em Administração) - Universidade Federal do Rio Grande do Sul, Porto Alegre, 2003.

LARAN, J.; ESPINOZA, F. Consumidores Satisfeitos, e Então? Analisando a Satisfação como Antecedente da Lealdade. Revista de Administração Contemporânea, v. 8, n. 2, p. 51-70, 2004.

LAU, G.; LEE, S. Consumers' trust in a brand and the link to brand loyalty. Journal of Market Focused Management. v. 4, p. 341-370, Dec., 1999.

LAWRANCE, R. Patterns of buyer behavior: time for a new approach? Journal of Marketing Research. v.6, p.137-144, may, 1969.

LEI, J.; PRUPPERS, R.; OUWERSLOOT, H.; LEMMINK, J. Service intensiveness and brand extension evaluations. Journal of Service Research, 
v. 6 , n. 3, p. 243-255, 2004.

LIANG, X.; ZHANG, S. Investigation of customer satisfaction in student food service An example of student cafeteria in NHH. International Journal of Quality and Service Sciences. v. 1. n. 1. p. 113124, 2009.

LIMA, F. C.; GAVA, R.; TAVARES, B. O processo de incubação como prestação de serviço: uma análise da percepção dos empresários sobre suas expectativas, satisfação e qualidade do serviço.ENCONTRO DE MARKETINF DA ANPAD, 3., 2008, Curitiba. Anais... Curitiba: EMA, 2008.

LOPES, H.; ALVES, R.; LEITE, R. Qualidade Percebida em Serviços: Um Estudo da Influência do Perfil Discente numa Instituição de Ensino Superior por Meio da Regressão Ordinal e da Análise de Aglomerados Two-Step. ENCONTRO DE MARKETING DA ANPAD, 3., 2008, Curitiba. Anais... Curitiba: EMA, 2008.

LOPES, E.; NOHARA, J.; HERNANDEZ, J.; ACEVEDO, C. Avaliação do Impacto da Qualidade de Serviços na Lealdade dos Consumidores de Materiais para Construção Civil: Aplicação da Escala RSQ Nacionalizada em Homecenters. ENCONTRO DE MARKETING DAANPAD, 3., 2008, Curitiba. Anais... Curitiba: EMA, 2008.

LOVELOCK, C.; WIRTZ, J. Marketing de Serviços: pessoas, Tecnologia e Resultados. Pearson Prentice Hall, 2006.

MACHADO, F. Um Estudo Sobre os Níveis de Fidelidade do Consumidor em Serviços de Naturezas Distintas. ENCONTRO DA ANPAD, 28., 2004, Curitiba. Anais... Curitiba: EnANPAD, 2004.

MARCCHETTI, R.; PRADO, P.; CUPERSCHMID, N. Proposição e teste de um modelo para avaliação da qualidade do relacionamento em serviços de distribuição de gás. ENCONTRO DE MARKETING DAANPAD, 2., 2006, Rio de Janeiro. Anais... Rio de Janeiro: EMA, 2006.

MASTERSON, S. ; LEWIS, K.; GOLDMAN, B.; TAYLOR, M. Integrating Justice and Social Exchange: The Differing Effects of Fair Procedures and Treatment on Work Relationships. Journal of the Academy of Management, v. 43, n. 4 p. 738-748, 2000.

MATHWICK, C.; MALHOTRA, N.; RIGDON, E. Experiential value: conceptualization, measurement and application in the catalog and Internet shopping environment. Journal of Retailing, Spring, v. 77, p. 39-56, 2001.

MCCRACKEN, G. Cultura \& Consumo. Rio de Janeiro: Mauá, 2003.

MONROE, K.; KRISHNAN R. The effect of price on subjective product evaluations. In: . Perceived quality: how consumers view stores and merchandise. Lexington: Lexington Books, 1985. p. 209-232.

MOORMAN, C.; DESHPANDÉ, R.; ZALTMAN, G. Factors Affecting Trust in Marketing Research Relationships. Journal of Marketing, v. 57, p. 81101, 1993.

MOORMAN, C.; ZALTMAN, G.; DESHPANDÉ, R. Relationships Between Providers and Users of Market Research: The Dynamics of Trust Within and Between Organizations. Journal of Marketing Research, v. 29, 1992.

MORGAN, R.; HUNT, S. The commitment-Trust Theory of Relationship Marketing. Journal of Marketing, v. 58, p. 20-38, 1994

MOWEN, J.; MINOR, M. Comportamento do consumidor. São Paulo: Prentice-Hall, 2003. 
NEAL, W. Satisfaction is nice, but value drives loyalty. Marketing Research. v.11, p.20-23, Spring, 1999.

NELSON, P. Advertising as Information. Journal of Political Economy, v.. 82, n. 4, p. 729-754, 1974.

NEWMAN, J. W.; WERBEL, R. A. Multivariate Analysis of Brand Loyalty for Major Household Appliances. Journal of Marketing Research, v. 10, n. 4; p. 404-410, 1973.

NIJSSEN, E.; SINGH, J.; SIRDESHMUKH, D.; HOLZMÜELLER, H. Investigating industry context effects in consumer-firm relationships: preliminary results from a dispositional approach. Journal of Academy of Marketing Science. v.31, p. 46-60, Winter, 2003.

OAKES, G. The Sales Process and the Paradoxes of Trust. Journal of Business Ethics, v. 9, n. 8, p. 671, 1990.

OLIVER, R. Acognitive model of the antecedents and consequences of satisfaction decisions. Journal of Marketing Research, v. 17, p. 460-469, 1980.

OLIVER, R. Cognitive, affective and attribute bases of satisfaction response. Journal of Consumer Research, v. 20, p. 418-430, 1993.

OLIVER, R. Whence consumer loyalty. Journal of Marketing. v.63, p. 33-44, Oct., 1999.

OLSHAVSKY, R.; KUMAR, A. Revealing the Actual Roles of Expectations in Consumer Satisfaction with Experience and Credence Goods. Journal of Consumer Satisfaction, Dissatisfaction and Complaining Behavior, v. 14, p. 60-73, 2001.

PARASURAMAN, A.; ZEITHAML, V. A.; BERRY, L.L. (1985) A conceptual model of service quality and its implication for future research. Journal of Marketing. Chicago, v.49, n.4, p. 41-51, 1985.
PARASURAMAN, A.; ZEITHAML, V. A.; BERRY, L.L. Servqual: a multiple-item scale for measure consumer perceptions of service quality. Journal of Retailing, v.64, n., p. 12-41, 1988.

PEREZ, C. Signos da marca: Expressividade e sensorialidade. São Paulo: Thomson Learning, 2004.

PEREZ, C. Universo Signico do Consumo: o sentido das marcas. Tese (Livre-Docência) São Paulo. Escola de Comunicação e Artes - ECAUSP, 2007.

PERIN, M. G.; SAMPAIO, C. H.; BREI, V. A.; PORTO, C. A. As Relações entre Confiança, Valor e Lealdade: um Estudo Intersetorial. ENCONTRO DA ANPAD, 28., 2004, Curitiba. Anais..., Curitiba; ENANPAD, 2004.

PINA, J.; MARTINEZ, E.; CHERNATONY, L.; DRURY, S. The effect of service brand extensions on corporate image: An empirical model. European Journal of Marketing, v. 40, n. 1-2, p. 174-197, 2006.

PRADO, P.; SANTOS, R. Comprometimento e Lealdade: Dois Conceitos ou Duas Dimensões de um Único Conceito? ENCONTRO DA ANPAD, 27., 2003, São Paulo. Anais... São Paulo: ENANPAD, 2003.

PRADO, P.; SANTOS, R. Satisfação, qualidade no relacionamento e lealdade entre clientes e banco de varejo.ENCONTRO DE MARKETING DA ANPAD, 1., 2004, Porto Alegre. Anais... Porto Alegre: EMA, 2004.

PRADO, P. H. M. A avaliação do relacionamento sob a ótica do cliente: um estudo em bancos de varejo. São Paulo. Tese (Doutorado em Administração) - Programa de Pós-Graduação em Administração de Empresas. Escola de Administração de Empresas de São Paulo. Fundação Getúlio Vargas, 2004. 
PRITCHARD, M.; HAVITZ, M.; HOWARD, D. Analysing the Commitment-Loyalty Link on Service Contexts. Journal of the Academy of Marketing Science, Summer, v.27, p.333-348, 1999.

RAO, A.; QU, L.; RUEKERT, R. Signaling unobservable product quality through a brand ally. Journal of Marketing Research, v. 36, n. 2, p. 258268, 1999.

RIEL, A.; LEMMINK, J.; OUWERSLOOT, H. Consumer evaluations of service brand extensions. Journal of Service Research, v. 3, n.3, 220-231, 2001.

RODRIGUES, V. Qualidade Percebida dos Serviços Odontológicos sob a Perspectiva dos Clientes e dos Profissionais. ENCONTRO DE MARKETING DA ANPAD, 3., 2008, Curitiba. Anais... Curitiba: EMA, 2008.

ROSENBERG, L.; CZEPIEL, J. A marketing approach for customer retention. Journal of Consumer Marketing. v. 1, p. 45-21, 1983.

ROSSI, P.E.; BRAGA, S. P. A Satisfação dos clientes em relação aos serviços prestados por um organismo de Inspeção veicular. Revista Administração On Line, FECAP, v.5, n. 3, p.11-25, jul/set, 2004.

RUYTER, K.; WETZELS, M. The role of corporate image and extension similarity in service brand extensions. Journal of Economic Psychology, v. 21, p. 639-659, 2000.

SANTOS, C.; ROSSI, C. O impacto do gerenciamento de reclamações na confiança e na lealdade do consumidor. Revista deAdministração Contemporânea. v. 6, p. 49-73, maio/ago, 2002.

SCHREYÖGG, G.; HÖPFL, H. Theatre and Organization: editorial introduction. Organizational Studies, v. 25, n.5, 2004.
SEMENIK, R.; BAMOSSY, G. Princípios de marketing: uma perspectiva global. São Paulo: Makron Books, 1996.

SETH, J.; MiTTAL, B.; NEWMAN, B. Comportamento do cliente: indo além do comportamento do consumidor. São Paulo: Atlas, 2001.

SETH, J..; PARK, C. A theory of multidimensional brand loyalty. Advances in Consumer Research. v. 1, p. 449-459, 1974.

SHETH, J.; PARVATIYAR, A. Relationship Marketing in Consumer Markets: Antecedents and Consequences. Journal of the Academy of Marketing Science, v. 23, n. 4, 1995.

SIMMEL, G. Sociología: Estudios sobre las formas de socialización. Madrid: Alianza Editorial, 1977.

SIMMEL, G. Crise de la culture moderne. In:

Philosophie de la modernité II. Traduzido por JeanLouis Viellard-Baron.Paris: Payot, 1990. p.179-217.

SINGH, J.; SIRDESHMUKH, D. Agency and Trust Mechanisms in Relational Exchanges. Journal of the Academy Marketing Science, v. 28, p. 150-167, 2000 .

SIQUEIRA, R. P. Desenvolvimento e Validação de uma Escala de Qualidade para o Ensino Superior de Administração: Um Trabalho Empírico. ENCONTRO DE MARKETING DA ANPAD, 1., 2004, Porto Alegre. Anais... Porto Alegre: EMA, 2004.

SIRDESHMUKH, D.; SINGH, J.; SABOL, B. Consumer Trust, Value, and Loyalty in Relational Exchanges. Journal of Marketing, v. 66, n. 1, p. 15-37, 2002.

SIROHI, N.; MCLAUGHLIN, E.; WITTINK, D. A model of consumer perceptions and store loyalty 
Qualidade: uma revisão da literatura...

intentions for a supermarket retailer. Journal of Retailing, Summerv. 74, p.223-245, Summer, 1998.

SMITH, D.C.; PARK, C.W. The effects of brand extensions on market share and advertising efficiency. Journal of Marketing Research, v. 29, n. 3, p. 296-310, 1992.

SPRENG, R.; MACKENZIE, S.; OLSHAVSKY, R. A reexamination of the determinants of consumer satisfaction. Journal of Marketing, v. 60, n.3, p. 1532, 1996.

SRINIVASAN, S.; ANDERSON, R.; PONNAVOLU, K. Customer loyalty in e-commerce: an exploration of its antecedents and consequences. Journal of Retailing. v.78, p. 41-50, Spring, 2002.

STRYKER, S.; STATHAM A. Symbolic Interaction and Role Theory. In: LINDZEY, G.; ARONSON, E. (Eds.) Handbook of Social Psychology. 3. ed. New York: Random House, 1985.

SWAMINATHAN, V.; FOX, R.J.; REDDY, S.K. The impact of brand extension introduction on choice. Journal of Marketing, v. 65, p. 1-15, 2001.

SWAN, J. E.; NOLAN, J. K. Gaining Customer Trust: A Conceptual Guide for the Salesperson. Journal of Selling and Sales Management, v. 5, p. 39-38, 1985.

TAYLOR, S.; BAKER, T. An assessment of the relationship between service quality and customer satisfaction in the formation of consumers' purchase intentions. Journal of Retailing. Summer, v. 70, p.163-178, 1994.

TEAS, K.R.; AGARWAL, S. The effects of extrinsic product cues on consumers' perceptions of quality, sacrifice, and value. Journal of the Academy of Marketing Science, v.28, n.2, p. 278-290, 2000.
THOMAS, D. Deluxe. Rio de Janeiro: Campus, 2008. TINOCO, M. A.; RIBEIRO, J. L. Uma nova abordagem para a modelagem das relações entre os determinantes da satisfação dos clientes de serviços. Revista Produção, São Paulo, v. 17, n. 3, 2007.

TINOCO, M.; PEREIRA, S.; RIBEIRO, J. D. Modelo de satisfação e atributos da qualidade para serviços de farmácia de manipulação. ENCONTRO DE MARKETING DAANPAD, 2010, Florianópolis. Anais... Florianópolis: EMA, 2010.

TSIROS, M.; MITTAL, V.; ROSS, W. The Role of Attributions in Customer Satisfaction: AReexamination. Journal of Consumer Research, v. 31, n. 2, p. 476 - 483, Sep. 2004.

TUCKER, W. The Development of Brand Loyalty. Journal of Marketing Research. v.1, p.32-35, Aug., 1964.

URDAN, A.;ZUÑIGA, M. Satisfação com assistência técnica e lealdade ao fabricante no ramo automobilístico. Revista de Administração de Empresas, v.41, p.31-41, jul./set., 2001.

VAN RAAIJ, W. F. Economic psychology. Journal of Economic Psychology, n. 1, p. 01-24, Mar. 1981.

VAKRATSAS, D., AMBLER, T. How Advertising Works: What do We Really Know? Journal of Marketing, v. 63, Jan., 1999.

WÄRNERYD, K.E. The psychology of innovative entrepreneurship. In W.F. van Raaij, G.M. van Veldhoven, \& K.E. Wärneryd (Eds.), Handbook of economic psychology. Dordrecht, Netherlands: Kluwer, 1988. p. 404-447.

VERHOEF, P.; FRANSES, P.; HOEKSTRA, J. The effect of relational constructs on customer referrals and number of services purchased from a multiservice provider: Does age of relationship matter? Journal of 
Academy of Marketing Science. v.30, p.202-216, Summer, 2002.

VIEIRA, V. A.; DAMACENA, C. Loyalty in the Supermarket. Brazilian Administration Review, v. 4, n. 3, p. 47-62, 2007.

VIEIRA, V. A.; TORRES, C. V. Verificação do Instrumento E-S-Qual para Mensuração da Qualidade Eletrônica no Varejo. ENCONTRO DE MARKETING DA ANPAD, 3., 2008, Curitiba. Anais... Curitiba: EMA, 2008.

VLACHOS, P.; VRECHOPOULOS, A. Determinants of behavioral intentions in the mobile internet services market. Journal of Services Marketing. v. 22, n. 4, p. 280-291, 2008.

VÖLCKNER, F.; SATTLER, H. Drivers of brand extension success. Journal of Marketing, v. 70, p. 18-34, 2006.

WHITTAKER, G.; LEDDEN, L.; KALAFATIS, S. A re-examination of the relationship between value, satisfaction and intention in business services. Journal of Services Marketing. v. 21, n. 5. p. 345-357, 2007.

WIND, Y; FRANK, R. Interproduct household loyalty to brands. Journal of marketing research. v. 6, p. 434-435, nov., 1969.

WOODALL, T. Conceptualising value for the customer: an attributional, structural and dispositional analysis. Academy of Marketing Science Review. Vancouver, v. 1, n. 12, p. 1-35, 2003.
XAVIER, W.; MARTINS, G.; LIMA, A.; GAVA, R.; TAVARES, B. O processo de incubação como prestação de serviço: uma análise da percepção dos empresários sobre suas expectativas, satisfação e qualidade do serviço. ENCONTRO DE MARKETING DAANPAD, 2., 2006, Rio de Janeiro. Anais... Rio de Janeiro: EMA, 2006.

YI, Y. A Critical review of consumer satisfaction. In:ZEITHAML, V. (ed.). Review of Marketing, 1990. p. 68-123.

YU, Y.; DEAN, A. The contribution of emotional satisfaction to consumer loyalty. International Journal of Service Industry Management. v. 12, p.234250, Feb., 2001.

ZEITHAML,V.A., Consumer perceptions of price, quality, and value: a means-end model and synthesis of evidence. Journal of Marketing, v.52, n.7, p.222, 1988.

ZEITHAML, V. ; BERRY, L. ; PARASURAMAN, A. The behavioral consequences of service quality. Journal of Marketing. v. 60, p. 31-46, Apr., 1996.

ZEITHAML, V.; BITNER, M. J.; GREMLER, D. Marketing de serviços: a empresa com foco no cliente. 5.ed. Porto Alegre: Ed. Bookman, 2011.

ZEITHAML, V.; PARASURAMAN, A.; BERRY, L. Delivering Quality Service; Balancing Customer Perceptions and Expectations. New York: Free Press, 1990. 